\title{
Monofrequency waveform acquisition and inversion: a new paradigm Tariq Alkhalifah, KAUST
}

\section{SUMMARY}

In seismic inversion, we tend to use the geometrical behavior of the wavefield (the kinematics), extracted from the data, to constrain the long wavelength model components and use the recorded reflections to invert for the short wavelength features in a process referred to as full waveform inversion (FWI). For such a recipe, single frequency (the right frequency) data are capable of providing the ingredients for both model components. A frequency that provides model wavelengths (through the transmission components) low enough to update the background and high enough (reflections) to map the scattering may render the other frequencies almost obsolete, especially large offset data are available to provide the transition from background to scattering components. Thus, I outline a scenario in which we acquire dedicated mono frequency data, allowing for more time to inject more of that single frequency energy at a reduced cost. The cost savings can be utilized to acquire larger offsets, which is an important for constraining the background model. Combing this single frequency data with a hierarchical scattering angle filter strategy in FWI, and potentially reflection FWI, provides an opportunity to invert for complex models starting even with poor initial velocity models. The objective of this new paradigm is a high resolution model of the Earth to replace our focus on the image, which requires a band of frequencies.

\section{INTRODUCTION}

Waveform inversion (FWI) typically requires a hierarchical approach from low to high frequency to maneuver the complex nonlinearity associated with the problem of velocity inversion (Bunks et al., 1995). The low frequency data admits low wavenumber updates of the velocity necessary to correct the background velocity model. The update process, however, suffers usually from the sinusoidal nature of wavefields, and the complexity of the inverted model yielding a highly non-linear relation (Lailly, 1983; Tarantola, 1984, 1986). Thus, any update in the model based on the iterative nature of the inversion could take us to a local minima (or maxima) model (Virieux and Operto, 2009). The hierarchical approach of full waveform inversion (FWI) of starting with the low frequencies and early arrivals part of the data is slowly giving way to more emphases on filtering and conditioning the gradient (Tang et al., 2013; Almomin and Biondi, 2013; Albertin et al., 2013). Such approaches are based on filtering the model update wavenumbers, which provides a separation of model update scales, and thus, emphasizing the transmission part of the wavefield first (Wu and Toksz, 1987; Mora, 1989). Alkhalifah (2014) demonstrated that low wavenumber updates are accessible more naturally through scattering angle filters applied to the gradients. This is true even for a single frequency, where high scattering angles (near transmission) information are inherently smeared over the model. In fact, all the information required to resolve a model at a reasonable resolution is buried in a frequency that produces wavelengths that manages to separate the geometrical and the scattering parts of the model update in an optimal way. In this case, the kinematic part of this wavefield is responsible for the low wavenumber components of the model and the reflections are responsible for the high wavenumber components. Ignoring the frequency redundancy nature of our wavefields has resulted in larger than needed acquisition and inversion costs.

In this abstract, I introduce a new paradigm in acquisition and inversion that complements the assertions made above in an effort to optimize our acquisition and inversion. Focussing on a single frequency in acquisition should yield more of the frequency energy at a reduced time and cost. The cost saving allows us to attain large offset data that will benefit from the enhanced energy of the monofrequency dominated wavefields. A frequency domain inversion using scattering angle filters allows us to control the model update wavenumber, and thus, maneuver the potential nonlinearity of the inversion. Synthetic examples demonstrate these features.

\section{THE MONOFREQUENCY CONCEPT}

The concept is based on the fact that our typical model of the Earth consists of long wavelength changes and very short wavelength interfaces. A frequency that produces wavelengths that are in between these two extremes (the background and the interface) can be used to resolve both the long wavelength information (from the geometrical ray embedded information in the wavefield) and the short wavelength information from the reflections in the data. A scattering angle filter developed by Alkhalifah (2014) can be used to guide us to the proper updates using mono frequency data.

The familiar handicap in FWI is in accessing the low and middle wavenumber information in the data. Some low wavenumber information may be provided by diving waves to some limited depth. So we end up utilizing geometrical based methods like tomography and MVA to obtain the low wavenumber information, and hopefully some of the middle wavenumber components. With the help of very low frequency data, we maybe able to fill the, much talked about, middle model wavenumber gap 


\section{Monofrequency inversion}

and achieve convergence in FWI. If the need for low frequencies is replaced by a scattering angle filter and the availability of large offsets, that gap might be filled with a single frequency. We always recognized that a single frequency (including the infinite one) is capable (under the right circumstances) of rendering an accurate smooth background velocity model. FWI shows us that the scattering component in our wavefield can provide us with interfaces at a higher resolution than what we obtain from imaging even if the frequency used to render the scattering is lower. This information is embedded in the amplitude behavior of the wavefield, which is ignored in imaging. Actually, the Helmholtz wave equation directly demonstrates that we can explicitly determine the velocity from the knowledge of the wavefield, $u(\mathbf{x}, \omega)$, at a single frequency.

\section{SINGLE FREQUENCY DATA}

Allowing our vibrators to sweep at a single frequency naturally boosts the energy of that frequency in the injected wavefields even if the sweep is short. We then record the mono frequency wavefield with listening devices (i.e. geophones) with a central frequency response corresponding to that frequency. To further isolate and enhance this frequency component, we cross correlate the data with this single frequency sweep. A single frequency, $\omega_{0}$, sweep can be represented by the following function: $f\left(\omega_{0}, t\right)=A(t) \sin \left(\omega_{0} t\right)$, where $t$ is the time variable and $A$ defines the amplitudes, which may include tapering to zero at the beginning and end of the sweep. The listening devices record the data, $d_{j}(t)$, where $j$ is an index corresponding to the receiver. As it is conventionally done in land acquisition, we crosscorrelate the sweep function with the recorded data as follows:

$$
d_{j}\left(\omega_{0}, t\right)=\int d_{j}(t+\tau) f\left(\omega_{0}, \tau\right) d \tau .
$$

This crosscorrelation process boosts the energy of the single frequency by approximately the length of the sweep. An alternative and equivalent application of the cross correlation is achieved by multiplying the data in the frequency domain with the complex conjugate of the Fourier representation of the source function. The final single frequency data is obtained using a single frequency Fourier transform:

$$
D_{j}\left(\omega_{0}\right)=\int_{0}^{t_{\max }} d_{j}\left(\omega_{0}, t\right) e^{-i \omega_{0} t} d t .
$$

This single frequency data is now ready for a frequency domain full waveform inversion. Before we do that, let us investigate what kind of data is obtained using such process. Figure 1(a) shows a real data trace borrowed from $\mathrm{Oz}$ Yilmaz Seismic wave Analysis book, in which the data is provided publicly and used here for demonstration. Figure 1(b) shows the frequency spectrum of the trace, with energy mainly falling between 5 and 40
$\mathrm{Hz}$. The crosscoleration of these data with a $10 \mathrm{~Hz}$ mono frequency time series of the same length as the trace (2.5 s) yields the frequency spectrum shown in Figure $1(\mathrm{c})$. Alternatively, using a time series that is $1 / 5$ the length of the trace in the cross correlation provides us with the spectrum shown in Figure 1(d). Clearly, the amplitude of the mono frequency signal increases when using a longer time series. In the frequency domain, it is equivalent to multiplying the amplitude at that frequency by the energy produced by the series. Nevertheless, the signal to noise ratio at that frequency does not change. However, the relation between the sweep length the amplitude is not linear, because it is controlled by the conservation of energy.

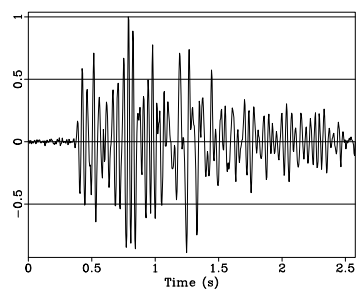

(a)

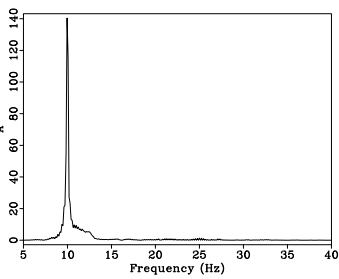

(c)

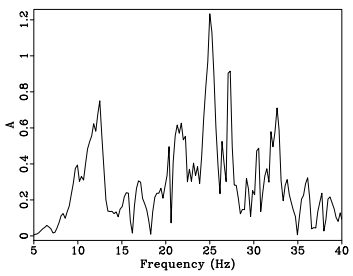

(b)

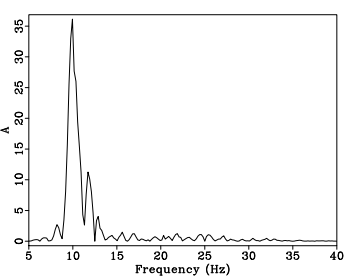

(d)
Figure 1: a) A real data trace borrowed from $\mathrm{Oz}$ Yilmaz Seismic wave Analysis book, b) It's amplitude spectrum, c) amplitude spectrum after cross correlating the trace with a $10 \mathrm{~Hz}$ mono frequency sweep of the same length as the trace, and d) amplitude spectrum after cross correlating the trace with a $10 \mathrm{~Hz}$ mono frequency sweep of one fifth that of the trace.

\section{FILTERING THE GRADIENT}

A direct approach for controlling the model wavenumber update is provided by filtering the gradient (Tang et al., 2013; Almomin and Biondi, 2013; Albertin et al., 2013). This recent development is based on the separation of scales in the update by applying the proper wavenumber filtering to the model update or matching the directional components of the source and receiver wavefields. A natural filter of the gradient capable of isolating the low wavenumber components of the update kernel is given by the scattering angle filter proposed by Alkhalifah (2014). It provides the required updates even for monochromatic wavefields

Introducing a slightly modified time lag (velocity scaled, 


\section{Monofrequency inversion}

$\zeta$ ) to our conventional gradient, $R$, (Khalil et al., 2013)

$$
R^{\prime}(\mathbf{x}, \zeta)=R\left(\mathbf{x}, \omega_{0}\right) e^{-4 i \omega_{0} \frac{\zeta}{v(\mathbf{x})}}
$$

where $v$ is the velocity, $\zeta=\frac{\tau}{2} v(\mathbf{x})$, and $\tau$ is the conventional time lag. An inherent feature of this modified time lag (distance units) representation is that the scattering angle relation to the wavenumber representation of the gradient is free of a velocity dependency, which has space dependence. In fact, the scattering angle, $\theta$, is given by the following formula:

$$
\cos ^{2} \frac{\theta}{2}=\frac{|\mathbf{k}|^{2}}{k_{\zeta}^{2}},
$$

where $\mathbf{k}$ is the wavenumber vector and $k_{\zeta}$ is the wavenumber (Fourier transform) corresponding to $\zeta$. So a four dimensional Fourier transform of $R^{\prime}(\mathbf{x}, \zeta)$ (three dimensional in $2 \mathrm{D})$, will allow us to map $\hat{R}\left(\mathbf{k}, k_{\zeta}\right)$ to it's angle gather equivalence, $\hat{R}(\mathbf{k}, \theta)$ using equation 4 . In our case, we use equation 4 to filter out the gradient energy corresponding to small $\theta$ (reflections), starting probably with $\theta<179$ degrees, and just sum the rest over $k_{\zeta}$ (the zero $\zeta$ imaging condition), no need to map to angle gathers. Of course, we will have to inverse Fourier transform the function back to space to apply the gradient in space. A scattering angle filter maintains the positive definite nature of the gradient function.

For minimum scattering angles of 100 and 160 degrees, the gray area in Figures 2(a) and 2(b), respectively, show the areas of the gradient represented in the $\zeta$ wavenumber domain that will survive the muting.

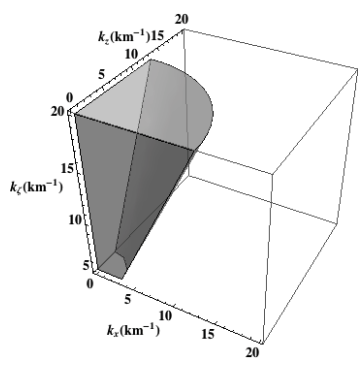

(a)

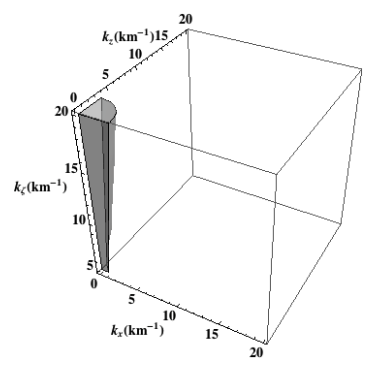

(b)
Figure 2: A plot showing in gray the regions in the model update (gradient) that will be spared the muting to only allow a minimum scattering angle of a) 100 and b) 160 degrees.

\section{MONOFREQUENCY POTENTIAL UPDATES}

We now consider the model shown in Figure 3(a), made up of a homogeneous box with a velocity of $5 \mathrm{~km} / \mathrm{s} \mathrm{em}$ bedded in a linearly increasing velocity model given by $v(z)=1.5+z \mathrm{~km} / \mathrm{s}$, where $z$ is depth. We consider an initial velocity model of $2 \mathrm{~km} / \mathrm{s}$, and test the ability of the gradient after filtering in predicting the perturbations in velocity for a mono frequency data of $4 \mathrm{~Hz}$. The data was acquired by placing the sources and receivers on the top boundary (the surface). Figure 3(b) shows the gradient computed using the adjoint state method. The mono frequency gradient yields, as expected, its largest energy near the reflection surface as the product of the state and adjoint state variables yields maximum values. It also has a sinusoidal nature reflecting the single, relatively high (in FWI world), frequency of the data. Applying a low cut scattering angle filter based on the described approach yields the gradient shown in Figures 3(c)-3(d). In all cases, we filtered out the high wavenumber components of the gradient to admit low wavenumber updates even in the direction normal to the reflector. Typically, we subtract the gradient (after proper scaling driven possibly by a line search) from the velocity model. Clearly, the filtered gradients reflect the need to decrease the velocity in the shallow part of the model to correct for the high velocity up shallow in the initial model. Specifically, the 179 degree filtered gradient admits a decrease up shallow and an increase deeper, which is in the general low wavenumber accurate direction. Though the increase deeper is mild, an application of an approximate Hessian (not applied here) might improve the weight of the deeper part as the Hessian diagonal elements are responsible for proper scaling to correct for geometrical spreading.

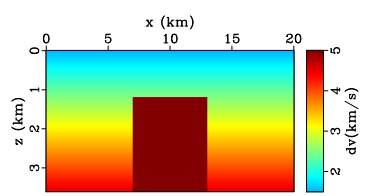

(a)

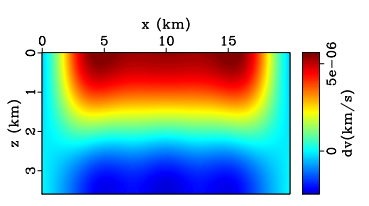

(c)

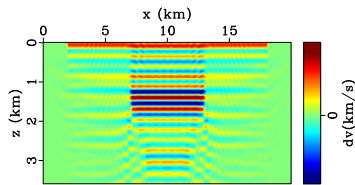

(b)

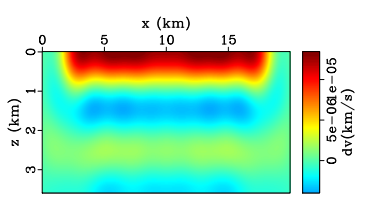

(d)
Figure 3: a) The true velocity model; b) The gradient corresponding to a constant background velocity of 1.5 $\mathrm{km} / \mathrm{s}$ and monochromatic wavefields of $4 \mathrm{~Hz}$ frequency. The updates after applying the low cut scattering angle filter muting angles below c) 179, and d) 178 .

\section{FREQUENCY VERSUS OFFSET}

Thanks to Wu and Toksz (1987), Mora (1989), and Sirgue and Pratt (2004) among others, we started to appreciate the role that large offsets play in improving FWI convergence. Like low frequencies, they provide low wavenumber updates. However, unlike low frequencies, large offsets provide zero model wavenumber updates for practical acquisition parameters. This is reflected in 


\section{Monofrequency inversion}

the model wavenumber formula given by

$$
\mathbf{k}^{\delta v}=\frac{\omega}{v} \cos \frac{\theta}{2} \mathbf{n}
$$

where $\mathbf{n}$ is a unit vector in the direction normal to a potential reflector. To obtain zero (or near zero) wavenumber updates in all directions we have two possibilities: low frequencies (near zero), or diving waves. The depth penetration of diving waves in the predominantly velocity increasing Earth is often proportional to the maximum offset recorded. The middle model wavenumber gap is often bordered from the top by the minimum frequency available, and from the bottom by the velocity resolution we manage to obtain from diving waves and/or MVA. Using a single frequency in acquisition allows us the latitude to increase the offset for two reasons:

- Some of the cost and time savings of a mono frequency acquisition can be utilized to acquire large offsets.

- The opportunity to inject more mono frequency energy allows for better detection of such energy at large offsets.

\section{WHAT FREQUENCY?}

Since the model update is mainly based on subtracting the gradients from the model, the maximum model wavenumber is limited to $k_{\max }=\frac{\omega_{0}}{v(\mathbf{x})}$. Thus, if the frequency is high, we may be able to obtain high resolution. Actually, infinite frequency, though not practical, is optimal. However, the higher the frequency the more complex the objective function becomes. To offset this complexity, as previously mentioned, we will need larger offsets. Well, this is the case in ideal conditions, specifically, no attenuation. Higher frequencies also suffer from larger dispersion losses. The more energy we are able to inject, considering the mono frequency nature of our source, the more energy we will be able to record, in spite of attenuation, but to a limit. Thus. the right frequency (or frequencies, maybe more than one) must combine the depth penetration requirements, with available the offsets, taking into consideration the attenuation factor.

\section{A MARMOUSI EXAMPLE}

Our next example is the famous benchmark Marmousi dataset (Versteeg, 1994). I only consider the $5 \mathrm{~Hz}$ frequency part of the data as if we only acquired that mono frequency. Figure 4(a) shows the true Marmousi model. Sources are considered every $100 \mathrm{~m}$ on the surface and receivers are located at all the grid points along the surface with a spacing of $25 \mathrm{~m}$. I use a homogeneous model $3 \mathrm{~km} / \mathrm{s}$ (Figure 4(b)) as the initial model for this single frequency FWI. The inversion, based on small steepest decent steps, starts at a low cut scattering angle of 179 degrees, followed by 178 , then $175,170,160$, and finaly 0 spending 20 iterations at each angle, and starting each angle with the velocity model we reached in the previous angle. The inverted velocity model at the end of scattering angle cut off of 178 and 170 are given in Figures 4(c) and 4(d), respectively. The final inverted velocity model is shown in Figure 4(e). Though the result is not perfect, considering the single (relatively high) frequency nature of the data, the inversion performed well. The result of using conventional FWI with no filtering for this $5 \mathrm{~Hz}$ data is shown in Figure $4(\mathrm{f})$.

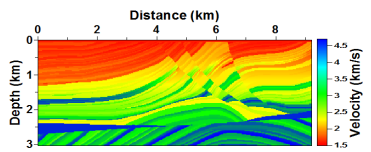

(a)

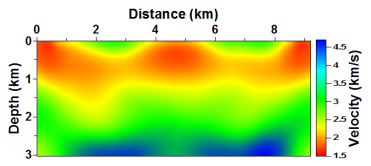

(c)

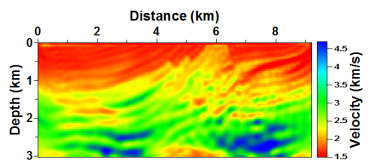

(e)

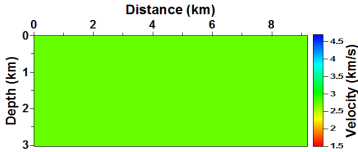

(b)

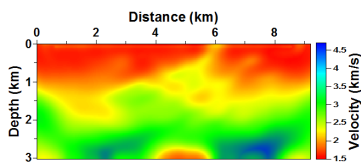

(d)

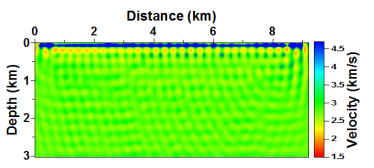

(f)
Figure 4: a) The Marmousi velocity model; b) The initial velocity model; c) The velocity at the end of using the low-cut scattering angle of 178 degrees; d) The velocity at the end of using the low-cut scattering angle of 170 degrees; e) The final result for this frequency; f) The result of conventional FWI (no filtering to the gradient).

\section{CONCLUSION}

Considering the typical Earth model of smooth layers separated by interfaces, a single frequency multi sources and receivers data include all the information needed to resolve almost all components of such a model. Thus, if we focus our acquisition and processing on mono frequency data, we can obtain more energy of that frequency at larger offsets at a reduced cost compared to conventional processing. The new filtering takes the ray information and smears it over the model domain, which allows the relatively high frequency data to yield low model wavenumber updates. Applications to synthetic data demonstrates such capabilities.

\section{ACKNOWLEDGMENTS}

I thank Yunseok Choi for generating some of the numerical examples. I thank KAUST for it's support. 
http://dx.doi.org/10.1190/segam2014-0836.1

\section{EDITED REFERENCES}

Note: This reference list is a copy-edited version of the reference list submitted by the author. Reference lists for the 2014 SEG Technical Program Expanded Abstracts have been copy edited so that references provided with the online metadata for each paper will achieve a high degree of linking to cited sources that appear on the Web.

\section{REFERENCES}

Albertin, U., G. Shan, and J. Washbourne, 2013, 2013, Gradient orthogonalization in adjoint scatteringseries inversion: $83^{\text {rd }}$ Annual International Meeting, SEG, Expanded Abstracts, doi: 10.1190/segam2013-0580.1.

Alkhalifah, T., 2014, Scattering angle base filtering of the FWI gradients: Presented at the $76^{\text {th }}$ Annual InternationalConference and Exhibition, EAGE.

Almomin, A., and B. Biondi, 2013, Tomographic full-waveform inversion (TFWI) by successive linearizations and scale separations : Presented at the $83^{\text {rd }}$ Annual International Meeting, SEG, Expanded Abstracts, 1048-1052.

Bunks, C., F. Saleck, S. Zaleski, and G. Chavent, 1995, Multiscale seismic waveform inversion: Geophysics, 60, 1457-1473, http://dx.doi.org/10.1190/1.1443880.

Khalil, A., J. Sun, Y. Zhang, and G. Poole, 2013, RTM noise attenuation and image enhancement using time-shift gathers: $83^{\text {rd }}$ Annual International Meeting, SEG, Expanded Abstracts, doi: 10.1190/segam2013-0600.1.

Lailly, P., 1983, The seismic inverse problem as a sequence of before-stack migrations, in Conference on Inverse Scattering: Theory and applications: SIAM, 206-220.

Mora, P., 1989, Inversion = migration + tomography: Geophysics, 54, 1575-1586, http://dx.doi.org/10.1190/1.1442625.

Sirgue, L., and R. Pratt, 2004, Efficient waveform inversion and imaging: A strategy for selecting temporal frequencies: Geophysics, 69, 231-248, http://dx.doi.org/10.1190/1.1649391.

Tang, Y., S. Lee, A. Baumstein, and D. Hinkley, 2013, Tomographically enhanced full-wavefield inversion: $83^{\text {rd }}$ Annual International Meeting, SEG, Expanded Abstracts, doi: 10.1190/segam20131145.1.

Tarantola, A., 1984, Inversion of seismic reflection data in the acoustic approximation: Geophysics, 49, 1259-1266, http://dx.doi.org/10.1190/1.1441754.

Tarantola, A., 1986, A strategy for nonlinear elastic inversion of seismic reflection data: Geophysics, 51, 1893-1903, http://dx.doi.org/10.1190/1.1442046.

Versteeg, R., 1994, The Marmousi experience: Velocity model determination on a synthetic complex data set: The Leading Edge, 13, 927-936, http://dx.doi.org/10.1190/1.1437051.

Virieux, J., and S. Operto, 2009, An overview of full-waveform inversion in exploration geophysics: Geophysics, 74, no. 6, WCC1-WCC26, http://dx.doi.org/10.1190/1.3238367.

Wu, R.-S., and M. N. Toksöz, 1987, Diraction tomography and multisource holography applied to seismic imaging: Geophysics, 52, 11-25, http://dx.doi.org/10.1190/1.1442237. 